\title{
SCALES OF FUNCTIONS
}

\author{
P. ERDÖS, C. A. ROGERS and S. J. TAYLOR
}

(received 7 September 1959)

\section{Introduction}

We consider real-valued functions $f(x)$ which are defined for all sufficiently large real numbers $x$. In discussing the behaviour of such functions as $x \rightarrow+\infty$, it is useful to compare $f$ with the functions of some "comparison scale". The early work in this field was due to Du Bois-Reymond (see, for example, (2), (3)). This was elaborated by Hardy, (6), who was mainly concerned with what he calls the "logarithmico-exponential" scale of functions. This "scale of Hardy" may be defined as the smallest class $\mathscr{H}$ of functions $f$ with the following properties:

(i) if $f \in \mathscr{H}, f$ is defined and continuous for all sufficiently large values of $x$;

(ii) (a) the function $f(x) \equiv \alpha$, where $\alpha$ is any real constant, is in $\mathscr{H}$; (b) the function $f(x) \equiv x$ is in $\mathscr{H}$;

(iii) if $f$ and $g$ are in $\mathscr{H}$ and $g$ is non-zero for all sufficiently large values of $x$, then

are also in $\mathscr{H}$.

$$
t / g, \quad \exp t, \quad \log |g|
$$

Hardy proved the existence of such a class $\mathscr{H}$, and showed that every $f$ in $\mathscr{H}$ is monotonic for sufficiently large values of $x$. He further proved that the functions $f$ in $\mathscr{H}$ are differentiable for large $x$ and that $f^{\prime} \in \mathscr{H}$. The system $\mathscr{H}$ is essentially the system of elementary functions built up from a finite number of operations $(+,-, \times, \div, \exp , \log )$ and the functions specified in (ii) above ${ }^{1}$. For a recent account of the system $\mathscr{H}$, from an abstract point of view, see Bourbaki (1).

In recent work (7), some of us required a scale of functions $\mathscr{S}$ which had some of the properties of $\mathscr{H}$, but in addition had the property of being maximal, i.e. with the property that there is no function $f(x)$ defined for large values of $x$ such that, for each $g$ in $\mathscr{S}$, either $f / g \rightarrow 0$ or $f / g \rightarrow+\infty$ as $x \rightarrow+\infty$.

The existence of maximal scales can be proved without difficulty by using

1 For example $x^{\alpha}=\exp [\alpha /(1 / \log x)]$, and $x^{2}-x=\log [\exp \{\exp [2 /(1 / \log x)]\} / \exp x]$. 
Zorn's lemma (or the axion of choice). The proofs are given in section 3 . Clearly there will be more than one such maximal system, and the use of Zorn's lemma does not allow much control over the properties of the system. For example, the condition that $\mathscr{S}$ is maximal does not imply, either (A) that $\mathscr{S}$ contains functions which tend to $+\infty$ more rapidly than any given function, or (B) that $\mathscr{S}$ contains functions which tend to $+\infty$ more slowly than any given function. The problem which gave rise to this paper was that of deciding firstly whether all maximal scales have properties (A) and (B), and secondly whether there exists at least one maximal scale with these properties.

It is clear that the detailed structure of a maximal scale $\mathscr{S}$ will not become evident, unless some constructive method is used for its definition. We have been unable to obtain such a method without the use of the continuum hypothesis. However, in sections 5 and 6 we do assume that hypothesis and are able to deduce the existence of two maximal scales, one having the properties (A) and (B), and the other having neither of these properties.

In producing the maximal scale, we manage to preserve the conditions that, if $f$ and $g$ are in $\mathscr{S}$, then so is $f / g$, and that each function in $\mathscr{S}$ is ultimately monotonic. It is this last requirement of monotonicity which causes a lot of the difficulties in the argument. In section 4, we extend several of the known results about enumerable collections of functions to enable us to add single functions to such collections and still preserve the monotonicity requirements outlined above. The results of section 4 do not depend on the continuum hypothesis, and are of interest in themselves.

In section 2, we give precise definitions of the ideas outlined in this introduction, and describe the previous results in the field which are needed in the sequel.

In this paper, we restrict the discussion to the behaviour of real functions of the real variable $x$ as $x$ approaches infinity. It is clear that, by using the terminology of Bourbaki (1), the methods apply to the study of a much wider class of functions. In particular, precisely the same results are applicable to the discussion of the behaviour of real functions $f(x)$ as $x$ approaches $\alpha$ (any real constant) from the right or from the left.

It seems likely that all the properties (other than its minimal property) of the scale of Hardy could be built into our new scales $\mathscr{S}$. In the present paper, we have not attempted to do this: in particular, the functions of $\mathscr{S}$ are not restricted to be differentiable. As we proceed, the reader will realize that the details of the proof would become extremely formidable if an attempt were made to preserve all the properties of $\mathscr{H}$. 


\section{Definitions, notation, and previous results}

The class of real functions $f(x)$ which are positive and continuous for $x \geqq 0$ and satisfy ${ }^{2} f(0)=1$ will be denoted by $\mathscr{C}$.

Italic letters $e, f, g, t, s, u, \cdots$ will be used to denote members of $\mathscr{C} ; i$ denotes the function

$$
\begin{aligned}
& i(x)=1, \quad \text { for } 0 \leqq x \leqq 1, \\
& i(x)=x, \quad \text { for } x \geqq 1 ;
\end{aligned}
$$

$k$ denotes the function $k(x) \equiv 1$ for $x \geqq 0$. The variables $x, \xi$ will be restricted to non-negative real values. The early part of the Greek alphabet $\alpha, \beta$, $\gamma, \cdots$ will denote real numbers: the later part $\mu, \nu, \pi, \rho, \sigma, \tau, \cdots$ (apart from $\xi)$ will be reserved for ordinal numbers.

Two functions $f, g$ in $\mathscr{C}$ are said to be equivalent whenever the ratio $f(x) / g(x)$ tends to a finite positive limit as $x \rightarrow+\infty$. We then write $f \sim g$ : clearly $\sim$ is an equivalence relation in the class $\mathscr{C}$. If $f, g$ in $\mathscr{C}$ are such that $\lim _{x \rightarrow+\infty} f(x) / g(x)=0$, we say that $f$ is of smaller order than $g$ at plus infinity and write $f \prec g$, or $g \succ f$. The relation $\prec$ introduces a partial ordering into $\mathscr{C}$ (and, by implication, into the system of equivalence classes determined by $\sim)$. We say that two functions $f, g$ in $\mathscr{C}$ are comparable if

$$
f \prec g \text { or } f \sim g \text { or } f \succ g \text {; }
$$

and that they are monotonically comparable if, in addition, the ratio $f(x) / g(x)$ is a monotonic function of $x$ for all sufficiently large values of $x$. The formula $f \leq g$ will mean that either $f \prec g$ or $f \sim g$. The subset of $\mathscr{C}$, consisting of functions $f$ which tend to $+\infty$ as $x \rightarrow+\infty$, will be denoted $\mathscr{C}^{\infty}$; the subset, consisting of functions $f$ which tend to 0 as $x \rightarrow+\infty$, will be denoted $\mathscr{C}$.

A subset $\mathscr{S} \subset \mathscr{C}$ is called a scale of functions if it has the three properties:

$\left(\mathrm{P}_{1}\right)$ if $f, g \in \mathscr{S}$, then $f$ and $g$ are comparable;

$\left(\mathrm{P}_{\mathbf{2}}\right)$ the functions $k(x)=1, i(x)=\sup (1, x)$ are in $\mathscr{S}$;

$\left(\mathrm{P}_{3}\right)$ if $f, g \in \mathscr{S}$ and $\alpha, \beta$ are real numbers, then $f^{\alpha} g^{\beta} \epsilon \mathscr{S}$ [where $\left.f^{\alpha} g^{\beta}(x)=(f(x))^{\alpha}(g(x))^{\beta}\right]$.

The scale $\mathscr{S}$ is said to be a monotone scale if it has the additional property $\left(\mathrm{P}_{1}^{*}\right)$ if $f, g \in \mathscr{S}$, then $f$ and $g$ are monotonically comparable.

In the introduction, we discussed the scale of Hardy $\mathscr{H}$. It is clear that the functions in $\mathscr{H}$ need not lie in $\mathscr{C}$, but those that are ultimately positive can be modified for small values of $x$, without effecting their asymptotic properties, to ensure that they do lie in $\mathscr{C}$. It is clear that, provided the modification has been done sufficiently skilfully, this modified set $\mathscr{H}^{*}$ is a monotone scale in the sense of our definitions. Our scales $\mathscr{S}$ need not have the other properties

2 The condition $f(0)=1$ has been introduced for convenience in proofs; it clearly does not affect the behaviour of functions $f$ for large values of $x$. 
of $\mathscr{H}^{*}$; in particular, we do not require functions $f \in \mathscr{S}$ to be differentiable. However, we now introduce properties $\left(\mathrm{P}_{4}\right)-\left(\mathrm{P}_{7}\right)$ which seem desirable in a scale of functions but which are not satisfied by $\mathscr{H}^{*}$.

$\left(\mathrm{P}_{4}\right)$ If $\mathrm{f}, g \in \mathscr{S}$ and $f \neq g$, then $f$ is not equivalent to $g$.

A set $\mathscr{S}$ which has the property $\left(\mathrm{P}_{4}\right)$ is called irreducible. This is not a very important property of scales: our main reason for introducing $\left(\mathrm{P}_{\mathbf{4}}\right)$ is that proofs of theorems are easier to write out for scales with property $\left(\mathrm{P}_{4}\right)$ (the alternative is to consider the equivalence classes in $\mathscr{P}$ as the elements in a new scale $\mathscr{S}^{\prime}$ ).

$\left(\mathrm{P}_{5}\right)$ If $f \in \mathscr{C}$ and $f$ is comparable with each element of $\mathscr{S}$, then $f$ is equivalent to at least one element of $\mathscr{S}$.

A scale $\mathscr{S}$ which has the property $\left(\mathrm{P}_{5}\right)$ is called a maximal scale. One cannot adjoin an essentially different element of $\mathscr{C}$ to a maximal scale $\mathscr{S}$ without spoiling the property $\left(\mathrm{P}_{1}\right)$.

$\left(\mathrm{P}_{6}\right)$ If $g \in \mathscr{C}$, there is at least one $f$ in $\mathscr{S}$ such that $f \succ g$.

$\left(\mathrm{P}_{7}\right)$ If $g \in \mathscr{C}$ and $g \succ k$, there is at least one $f$ in $\mathscr{S}$ such that $g \succ t>k$.

Note that, since every $\mathscr{S}$ satisfies $\left(\mathrm{P}_{3}\right)$, the property $\left(\mathrm{P}_{6}\right)$ implies that, given any $g \in \mathscr{C}$, there exists an $f$ in $\mathscr{S}$ with $f \prec g$. Thus $\left(\mathrm{P}_{6}\right)$ expresses formally the idea that a scale is extensive in the sense that it contains functions which diverge to $+\infty$ and converge to 0 arbitrarily rapidly. Similarly $\left(\mathrm{P}_{\mathbf{7}}\right)$ implies that, if $g \in \mathscr{C}$ and $g \prec k$, there is at least one $f$ in $\mathscr{S}$ such that $g \prec f \prec k$. This, in turn, implies that, if $g$ in $\mathscr{C}$ and $h, u$ in $\mathscr{S}$ are such that $h \prec g \prec u$, then there exist functions $t, t$ in $\mathscr{S}$ such that $h \prec t \prec g \prec t \prec u$. Thus $\left(\mathrm{P}_{7}\right)$ expresses formally the idea that the scale has a fine structure, in the sense that the subset of $\mathscr{S}$, containing those functions which are asymptotically larger than (or smaller than) a given function of $\mathscr{C}$ (not necessarily in $\mathscr{S}$ ), has no least (or greatest) element.

A set $\mathscr{S}$, which has the properties $\left(\mathrm{P}_{6}\right)$ and $\left(\mathrm{P}_{7}\right)$, will be said to be dense. At first sight, one is tempted to think that the properties $\left(\mathrm{P}_{6}\right)$ and $\left(\mathrm{P}_{7}\right)$ might be a consequence of $\left(\mathrm{P}_{5}\right)$. With the use of the continuum hypothesis, we show, (i) in section 5 , that there exist monotone scales with properties $\left(\mathrm{P}_{4}\right)-\left(\mathrm{P}_{7}\right)$, and, (ii) in section 6 , that there exist monotone scales with properties. $\left(P_{4}\right)$ and $\left(P_{5}\right)$ but for which $\left(P_{6}\right),\left(P_{7}\right)$ are false. Since the continuum hypothesis is known to be not inconsistent with the usual axioms of mathematics, this shows that it is impossible to prove that a scale which is maximal must also be dense. One can similarly show that $\left(\mathrm{P}_{6}\right)$ and $\left(\mathrm{P}_{7}\right)$ are independent of each other.

In the sequel we shall need

LEMma 1. Given a sequence of functions $f_{1} \prec f_{2} \prec f_{3} \prec \cdots \prec f_{n} \prec \cdots$ in 
$\mathscr{C}$, there exists a function $f$ in $\mathscr{C}$ such that $f>f_{n}$ for each positive integer $n$.

This is due to du Bois-Reymond (2). The proof is simple: take $f(x)=$ $1+\sum_{r \leqq x}(x-r) f_{r}(x)$.

LEMMA 2. (i) Given a sequence of functions $f_{1} \prec f_{2} \prec f_{3} \prec \cdots \prec f_{n} \prec \cdots$, and a function $f$ in $\mathscr{C}$ such that $f_{n} \prec f$ for each integer $n$, there exists a function $g$ in $\mathscr{C}$ such that $f_{n} \prec g \prec f$ for each integer $n$.

(ii) Given a sequence of functions $f_{1} \succ f_{2}>f_{3} \succ \cdots>f_{n} \succ \cdots$, and a function $f$ in $\mathscr{C}$ such that $f_{n}>f$ for each integer $n$, there exists a function $g$ in $\mathscr{C}$ such that $f_{n}>g>f$ for each integer $n$.

This is due to Hadamard (4).

LEMMA 3. If $\mathscr{L}$ is any partially ordered set, then there is a maximal subset $\mathscr{M} \subset \mathscr{L}$ which is simply ordered (i.e. $\mathscr{K}$ is not a proper subset of any simply ordered $\mathscr{M}^{\prime} \subset \mathscr{L}$ ).

This is the form we need of the celebrated Zorn's lemma which is known to be equivalent to the axiom of choice or the well ordering principle.

\section{Existence of maximal scales}

The main result of this section is

THEOREM 1. There exists a scale of functions $\mathscr{S}$, which is irreducible and maximal.

To prove the theorem, we have to show the existence of a subset $\mathscr{S} \subset \mathscr{C}$ with the properties $\left(\mathrm{P}_{1}\right)-\left(\mathrm{P}_{5}\right)$. Let $\mathscr{T}$ denote the collection of those subsets $\mathscr{U} \subset \mathscr{C}$ which have the four properties $\left(\mathrm{P}_{1}\right)-\left(\mathrm{P}_{4}\right)$. Then $\mathscr{T}$ is not empty, since the class $\mathscr{U}$ of all functions of the form $\{i(x)\}^{\alpha}$, for real $\alpha$, is a member of $\mathscr{T}$. Further, $\mathscr{T}$ is partially ordered by the relation of inclusion, i.e. we say that $\mathscr{U}_{1} \leqq \mathscr{U}_{2}$ if $\mathscr{U}_{1} \subset \mathscr{U}_{2}$. Use lemma 3 (Zorn's lemma) to obtain a system $\mathscr{V} \subset \mathscr{T}$ which is simply ordered and maximal, in the sense that it is impossible to add a further class $\mathscr{U} \in(\mathscr{T}-\mathscr{V})$ to $\mathscr{V}$ and preserve the simple ordering. Now put $\mathscr{S}=\cup_{\mathscr{U} \in \mathscr{V}} \mathscr{U}$. By using the fact that, if $\mathscr{U}_{1}, \mathscr{U}_{2} \in \mathscr{V}$ then either $\mathscr{U}_{1} \subset \mathscr{U}_{2}$ or $\mathscr{U}_{2} \subset \mathscr{U}_{1}$, it is easy to verify that the subset $\mathscr{S} \subset \mathscr{C}$ has the properties $\left(\mathrm{P}_{1}\right)-\left(\mathrm{P}_{4}\right)$. It only remains to show that $\mathscr{S}$ is maximal in the sense that it has property $\left(\mathrm{P}_{5}\right)$.

If $\mathscr{S}$ does not satisfy $\left(\mathrm{P}_{5}\right)$, there is a function $t$ in $\mathscr{C}$ which is comparable with every $f$ in $\mathscr{S}$ but not equivalent to any $f$ in $\mathscr{S}$. Let $\mathscr{S}(t)$ be the subset of $\mathscr{C}$ consisting of those elements of the form $t^{\alpha} f$ where $\alpha$ is any real number and $f \in \mathscr{S}$. The set $\mathscr{S}(t)$ clearly has properties $\left(\mathrm{P}_{2}\right)$ and $\left(\mathrm{P}_{3}\right)$. Consider any two different functions $t^{\alpha} f_{1}, t^{\beta} f_{2}$ in $\mathscr{S}(t)$. If $\alpha=\beta$ the functions are comparable but not equivalent, while if $\alpha \neq \beta$, we have

$$
\begin{aligned}
\frac{t^{\alpha} f_{1}}{t^{\beta} f_{2}} & =\left[t f_{1}^{1 /(\beta-\alpha)} f_{2}^{1 /(\alpha-\beta)}\right]^{\beta-\alpha} \\
& =\left[t / f_{3}\right]^{\beta-\alpha}, \text { for some } f_{3} \text { in } \mathscr{S},
\end{aligned}
$$


and again the functions $t^{\alpha} f_{1}, t^{\beta} f_{2}$ are comparable but not equivalent. Hence $\mathscr{S}(t)$ has properties $\left(\mathrm{P}_{4}\right)$ and $\left(\mathrm{P}_{1}\right)$. Consequently $\mathscr{S}(t) \in \mathscr{T}$ and contains as a proper subset every set of the system $\mathscr{V}$. This contradicts the definition of $\mathscr{V}$, and proves that $\mathscr{S}$ must satisfy $\left(\mathrm{P}_{5}\right)$. This completes the proof of the tieorem.

It is clear that the method of proof of theorem 1 can be applied to monotone irreducible scales, that is, sets $\mathscr{S} \subset \mathscr{C}$ which satisfy $\left(\mathrm{P}_{1}^{*}\right)$ and $\left(\mathrm{P}_{2}\right)-\left(\mathrm{P}_{4}\right)$. This leads to the idea of a maximal monotone scale, defined as a set $\mathscr{S}$ with the properties $\left(\mathrm{P}_{1}^{*}\right),\left(\mathrm{P}_{2}\right),\left(\mathrm{P}_{3}\right)$ and in addition:

$\left(\mathrm{P}_{5}^{*}\right)$ if $f \in \mathscr{C}$ and $f$ is monotonically comparable with each element of $\mathscr{S}$, then $f$ is equivalent to some element of $\mathscr{S}$.

Thus we can obtain

THEOREM 1A. There exists a maximal monotone scale of functions which is irreducible.

It should be noted that $\left(\mathrm{P}_{5}^{*}\right)$ is weaker than $\left(\mathrm{P}_{5}\right)$ when applied to scales satisfying $\left(\mathrm{P}_{1}^{*}\right)$. One of the objectives of this paper is to show the existence of scales $\mathscr{S}$ satisfying $\left(\mathrm{P}_{1}^{*}\right)$ and $\left(\mathrm{P}_{2}\right)-\left(\mathrm{P}_{5}\right)$. It does not seem that Zorn's lemma can achieve this end because, when more stringent conditions are imposed, maximality in the sense of Zorn's lemma becomes weaker ${ }^{3}$. There is an additional reason why we cannot use Zorn's lemma to obtain scales with properties $\left(\mathrm{P}_{6}\right)$ and $\left(\mathrm{P}_{7}\right)$ : the real difficulty here is to show the existence of one such scale. This explains why, in the rest of the paper, we need to use more constructive methods.

\section{Adding a single function}

The results of lemmas 1,2 refer to enumerable sets of functions of $\mathscr{C}$ which satisfy $\left(\mathrm{P}_{1}\right)$. The first step is to modify these so that, when $\left(\mathrm{P}_{1}^{*}\right)$ is true, it remains true of the set enlarged by the addition of a new function (the latter chosen to satisfy additional requirements). We shall need the following rather special lemma which does not seem to follow easily from known results:

LEMMA 4. Let $f, g, h$ be functions of $\mathscr{C}$ satisfying $f \prec g, f \prec h$ and such that $f$ and $g$ are monotonically comparable; then there are functions $l$ and $u$ of $\mathscr{C}$ which are monotonically comparable with $f$ and $g$ and satisfy

$$
t \prec l \prec g \prec u, \quad f<l<h \prec u \text {. }
$$

Proof. The function $u$ defined by

$$
u(x)=(1+x) g(x) \sup _{0 \leqq \xi \leqq x} \frac{h(\xi)}{g(\xi)}, \quad \text { for all } x \geqq 0,
$$

- For example it is possible to demand that the ratio $f / g$ for any two functions $f, g \in \mathscr{S}$ be monotonic for all $x \geqq 0$. In this case maximality becomes very weak indeed, and Mr. A. Beck has pointed out to us that there is a countable cofinal sequence for any such scale. 
clearly satisfies the required conditions. To obtain $l$, we first define a function by

where

$$
v(x)=\gamma f(x) \inf _{\xi \geqq x} \frac{h(\xi)}{f(\xi)}, \text { for } x \geqq 0,
$$

$$
\gamma=\sup _{\eta \geq 0} \frac{f(\eta)}{h(\eta)}
$$

It is clear that $v(x)$ is continuous, that

$$
v(x) \leqq \gamma h(x), \text { for all } x>0,
$$

and that $f \prec v$, while $v / f$ tends monotonically to $+\infty$. Now define a function $l$ by the relation

Then

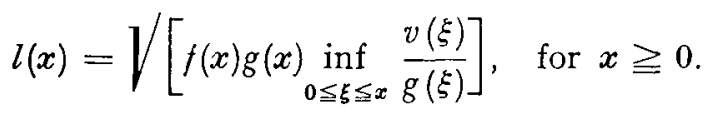

$$
\begin{aligned}
\left\{\frac{l(x)}{v(x)}\right\}^{2} & =\frac{f(x)}{v(x)} \cdot \frac{g(x)}{v(x)} \inf _{0 \leqq \xi \leqq x} \frac{v(\xi)}{g(\xi)} \\
& \leqq \frac{f(x)}{v(x)} \rightarrow 0 \quad \text { as } x \rightarrow+\infty .
\end{aligned}
$$

By (1) it follows that $l \prec h$. Further,

$$
\left\{\frac{l(x)}{g(x)}\right\}^{2}=\frac{f(x)}{g(x)} \inf _{0 \leqq \xi \leqq x} \frac{v(\xi)}{g(\xi)}
$$

and $\inf _{0 \leqq \xi \leq x} v(\xi) / g(\xi)$ does not exceed 1 , and is monotonic decreasing while $f(x) / g(x)$ decreases monotonically for $x \geqq X_{0}$, and tends to zero as $x \rightarrow \infty$. Hence $l \prec g$ and $l, g$ are monotonically comparable. It only remains to prove that $l \succ f$ and that $l, f$ are monotonically comparable.

Let $X_{1}$ be any fixed real number such that $g(x) \geqq f(x)$ for $x \geqq X_{1}$ and $g(x) / f(x)$ is monotonic increasing for $x \geqq X_{1}$. Let $X$ satisfy $X_{1} \leqq X \leqq x$. Then

$$
\begin{aligned}
\left\{\frac{l(x)}{f(x)}\right\}^{2} & =\frac{g(x)}{f(x)} \inf _{0 \leqq \xi \leqq x} \frac{v(\xi)}{g(\xi)} \\
& \geqq \min \left\{\frac{g(x)}{f(x)} \inf _{0 \leqq \xi \leqq X} \frac{v(\xi)}{g(\xi)}, \inf _{X \leqq \xi \leqq x} \frac{v(\xi)}{f(\xi)}\right\} .
\end{aligned}
$$

Since both $f \prec g$ and $f \prec v$, if we let $X \rightarrow+\infty$ slowly enough as $x \rightarrow+\infty$, we have $l \succ t$.

Suppose, if possible, that $l(x) / f(x)$ is not monotonic for $x \geqq X_{1}$. Let $x_{1}, x_{2}$ be such that $X_{1} \leqq x_{1}<x_{2}$ and 


$$
\frac{l\left(x_{1}\right)}{f\left(x_{1}\right)}>\frac{l\left(x_{2}\right)}{f\left(x_{2}\right)}
$$

Then, by (2), we would have

$$
\frac{g\left(x_{1}\right)}{f\left(x_{1}\right)} \inf _{0 \leqq \xi \leqq x_{1}} \frac{v(\xi)}{g(\xi)}>\frac{g\left(x_{2}\right)}{f\left(x_{2}\right)} \inf _{0 \leqq \xi \leqq x_{2}} \frac{v(\xi)}{g(\xi)} .
$$

Since

this implies that

$$
\frac{g\left(x_{1}\right)}{f\left(x_{1}\right)} \leqq \frac{g\left(x_{2}\right)}{f\left(x_{2}\right)}
$$

$$
\inf _{0 \leqq \xi \leqq x_{1}} \frac{v(\xi)}{g(\xi)}>\inf _{0 \leqq \xi \leqq x_{2}} \frac{v(\xi)}{g(\xi)} .
$$

Hence for some $\xi_{0}$ with $x_{1}<\xi_{0} \leqq x_{2}$, we have

Thus

$$
\inf _{0 \leqq \xi \leqq x_{2}} \frac{v(\xi)}{g(\xi)}=\frac{v\left(\xi_{0}\right)}{g\left(\xi_{0}\right)} \text {. }
$$

$$
\begin{aligned}
\frac{v\left(x_{1}\right)}{f\left(x_{1}\right)} & \geqq \frac{g\left(x_{1}\right)}{f\left(x_{1}\right)} \inf _{0 \leqq \xi \leqq x_{1}} \frac{v(\xi)}{g(\xi)} \\
& >\frac{g\left(x_{2}\right)}{f\left(x_{2}\right)} \inf _{0 \leqq \xi \leqq x_{2}} \frac{v(\xi)}{g(\xi)} \\
& \geqq \frac{g\left(\xi_{0}\right)}{f\left(\xi_{0}\right)} \inf _{0 \leqq \xi \leqq x_{2}} \frac{v(\xi)}{g(\xi)}=\frac{v\left(\xi_{0}\right)}{f\left(\xi_{0}\right)} .
\end{aligned}
$$

Since $v(x) / f(x)$ is monotonic increasing for all $x$, this is a contradiction which establishes that $l(x) / f(x)$ is monotonic for $x \geqq X_{1}$. This completes the proof of the lemma.

LEMmA 5. Suppose that $\mathscr{Z}$ is a countable subset of $\mathscr{C}$ such that any two functions of $\mathscr{Q}$ are monotonically comparable and that a function $f$ of $\mathscr{C}$ is comparable with each element of $\mathscr{Q}$. Then there exists a function $g$ in $\mathscr{C}$, with $f \prec g$, which is monotonically comparable with each element of $\mathscr{Q}$, and which satisfies

for every $q$ in 2 with $f<q$.

$$
g<q
$$

Proof. Let $q_{1}, q_{2}, \cdots$ be any enumeration of the functions of $\mathscr{2}$. Let $\mathscr{Q}^{+}$denote the set of functions $q$ of $\mathscr{Q}$ with $f<q$ and let $\mathscr{Q}^{-}$denote the set of functions $q$ of $\mathscr{2}$ with $q \prec f$. We consider separately the three cases when:

(i) $2^{+}$contains elements but contains no minimal element ${ }^{4}$;

- A function $q_{0}$ is said to be a minimal element of $\mathscr{Q}^{+}$if every element $q$ of $\mathscr{Q}^{+}$other than $q_{0}$ satisfies $q_{0} \leq q$. 
(ii) $2^{+}$contains a minimal element;

(iii) $\mathscr{Q}^{+}$is empty.

(i) In this case, we can choose a sequence $v_{1}, v_{2}, \cdots$ of elements of $\mathscr{2}^{+}$ such that $v_{1} \succ v_{2} \succ v_{3} \succ \cdots$ and such that each $q$ in $\mathscr{Q}^{+}$satisfies $q \succ v_{r}$ for all sufficiently large $r$. We define inductively a sequence of real numbers $\left\{\alpha_{r}\right\}$ and a sequence of functions $\left\{s_{r}\right\}$ by the conditions:

(i) (3) $\quad \alpha_{1}=0$, and $s_{1}(x)=v_{1}(x)$ for $x \geqq 0$;

(ii) for $r=1,2, \cdots$, if $\alpha_{r}, s_{r}$ are known we take $\alpha_{r+1}$ to be large enough to ensure that

(4) $\quad \alpha_{r+1}>\alpha_{r}+1$,

(5) that $s_{r}(x)>v_{r+1}(x)>r f(x)$ for $x \geqq \alpha_{r+1}$,

(6) that the ratio $v_{r+1}(x) / v_{r}(x)$ is monotonic for $x \geqq \alpha_{\tau+1}$,

(7) and that the ratios $v_{r+1}(x) / q_{s}(x), s=1,2, \cdots, r$, are all monotonic for $x \geqq \alpha_{r+1}$; and

(iii) we define $s_{r+1}(x)$ by

$$
\left\{\begin{array}{l}
s_{r+1}(x)=s_{r}(x) \text { for } 0 \leqq x \leqq \alpha_{r+1} \\
s_{r+1}(x)=\frac{s_{r}\left(\alpha_{r+1}\right)}{v_{r+1}\left(\alpha_{r+1}\right)} v_{r+1}(x) \text { for } \alpha_{r+1} \leqq x .
\end{array}\right.
$$

It is easy to check that this inductive definition can be carried out; the fact that $s_{r}(x)$ satisfies (8) with $r+1$ replaced by $r$ ensures that $\alpha_{r+1}$ can be chosen to satisfy the condition (5).

Now put $g(x)=\lim _{r \rightarrow \infty} s_{r}(x)$. Note that for any fixed $x$, we have

$$
s_{r}(x)=s_{r+1}(x)=s_{r+2}(x)=\cdots
$$

provided $\alpha_{r+1} \geqq x$. It follows from (6) and (8) that $g \prec v_{r}$ for each integer $r$, and therefore $g \prec q$ for all $q \in \mathscr{2}^{+}$. The conditions (5) and (8) ensure that $f<g$, while (7) and (8) ensure that $g$ is monotonically comparable with each element of $\mathscr{Q}$. Thus $g$ has the required properties.

(ii) Now suppose that $\mathscr{2}^{+}$contains at least one minimal element. Let $\mathscr{E}$ be the equivalence class of minimal elements of $\mathscr{Q}^{+}$. If the number of elements in $\mathscr{E}$ is finite, then $\mathscr{E}$ will contain an element $q_{+}$such that $q_{+}(x) / q(x)$ is monotonic decreasing for sufficiently large $x$ for each $q$ in $\mathscr{E}$ other than $q_{+}$. If $\mathscr{E}$ is infinite and does not contain an element $q_{+}$with this property, we must first adjoin such an element to $\mathscr{E}$, taking care that the new element is monotonically comparable with the rest of $\mathcal{Q}$.

Without loss of generality, we may assume that $\mathscr{E}$ is such that, if $q_{i}, q_{\text {s }}$ are two elements in $\mathscr{E}$, the ratio $q_{i}(x) / q_{j}(x) \rightarrow 1$ as $x \rightarrow+\infty$. If there is no element $q_{+}$with the required property, then there must be a sequence 
$t_{1}(x), t_{2}(x), \cdots, t_{k}(x), \cdots$ of functions of $\mathscr{E}$ such that, for $k=1,2, \cdots$,

$$
t_{k+1}(x)>t_{k}(x) \text { for sufficiently large } x \text {, }
$$

and for any $q$ in $\mathscr{E}, t_{k}(x) / q(x)$, for some integer $k$, is monotonic decreasing for large enough $x$. We define inductively a sequence of real numbers $\left\{\beta_{r}\right\}$ and a sequence of functions $\left\{s_{r}\right\}$ by the conditions

(i) $\beta_{1}=1, s_{1}(x)=t_{1}(x)$ for $x \geqq 0$;

(ii) for $r=1,2, \cdots$, if $\beta_{r}, s_{r}$ are known we take $\beta_{r+1}$ to be large enough to ensure that

$$
\beta_{r+1}>\beta_{r}+1
$$

that the ratio $t_{r+1}(x) / t_{r}(x)$ is monotonic and satisfies

$$
1+\frac{1}{r^{2}}>\frac{t_{r+1}(x)}{t_{r}(x)}>1 \text { for } x \geqq \beta_{r+1}
$$

and the ratios $t_{r+1}(x) / q_{s}(x), s=1,2, \cdots, r$ are monotonic for $x \geqq \beta_{r+1}$; and

(iii) we define $s_{r+1}(x)$ by

$$
\left\{\begin{array}{l}
s_{r+1}(x)=s_{r}(x) \text { for } 0 \leqq x \leqq \beta_{r+1} \\
s_{r+1}(x)=\frac{s_{r}\left(\beta_{r+1}\right)}{t_{r+1}\left(\beta_{r+1}\right)} t_{r+1}(x) \text { for } \beta_{r+1} \leqq x
\end{array}\right.
$$

Now put $q_{+}(x)=\lim _{r \rightarrow+\infty} s_{r}(x)$. The conditions (11) and (13) ensure that $q_{+}$is equivalent to each element of $\mathscr{E}$, and that $q_{+} / q$ is ultimately monotonic decreasing for each $q$ in $\mathscr{E}$, tending to the finite positive limit

$$
\prod_{r=1}^{\infty} \frac{t_{r}\left(\beta_{r+1}\right)}{t_{r+1}\left(\beta_{r+1}\right)}
$$

Conditions (12) and (13) ensure that $q_{+}$is monotonically comparable with every element of $\mathscr{Q}$.

Thus we may assume that there is a function $q_{+}$in $\mathscr{2}^{+}$with the property that $q_{+}(x) / q(x)$ is monotonic decreasing for sufficiently large $x$ for every $q$ in $2^{+}$other than $q_{+}$.

There are now three possibilities to consider:

(a) $2^{-}$is empty,

(b) $2^{-}$is not empty and contains no element $q_{-}$such that $q_{-} / q$ is ultimately monotonic increasing for each $g$ in $\mathscr{Q}^{-}$other than $q_{-}$,

(c) $\mathscr{Q}^{-}$is not empty and contains an element $q_{-}$such that $q_{-} / q$ is ultimately monotonic increasing for every $q$ in $\mathscr{Q}^{-}$other than $q_{-}$.

(a) Let $g(x)$ be defined by 


$$
g(x)=q_{+}(x)\left[\inf _{\xi \geqq x} q_{+}(\xi) / f(\xi)\right]^{-\frac{1}{2}}, \text { for } x \geqq 0 .
$$

Since $f \prec q_{+}$, the function

$$
\inf _{\xi \geqq x} q_{+}(\xi) / f(\xi)
$$

is monotonic increasing, and diverges to $+\infty$. Consequently $g$ and $q_{+}$are monotonicaly comparable and $g \prec q_{+}$. Hence $g$ is monotonically comparable with each function $q$ of $\mathscr{Q}$. Further, since

$$
\frac{g(x)}{f(x)}=\frac{q_{+}(x)}{f(x)}\left[\inf _{\xi \geqq x} \frac{q_{+}(\xi)}{f(\xi)}\right]^{-\frac{1}{2}} \geqq\left[\inf _{\xi \geqq x} \frac{q_{+}(\xi)}{f(\xi)}\right]^{\frac{1}{2}},
$$

we deduce that $f \prec g$, as required.

(b) If $2^{-}$contains no maximal element, we use the argument of (i) to adjoin an element $q_{-}$which is monotonically comparable with the elements of $\mathscr{Q}$ and satisfies $f \succ q_{-} \succ q$ for each $q$ in $\mathscr{2}^{-}$. On the other hand, if $\mathscr{Q}^{-}$contains a class $\mathscr{G}$ of maximal elements, we can use a similar argument to that used in obtaining $q_{+}$from the class $\mathscr{E}$, to obtain a function $q_{-}$with the property that $q_{-} / q$ is ultimately monotonic increasing for every $q$ in $\mathscr{Q}^{-}$other than $q_{-}$. This reduces case (b) to case (c).

(c) We have now got the situation $q_{-} \leq f \prec q_{+}$and $q_{-}, q_{+}$are monotonically comparable. Apply lemma 4 to obtain a function $g$, which is monotonically comparable with $q_{-}$and $q_{+}$, and which satisfies $q_{-} \prec g \prec q_{+}, f \prec g \prec q_{+}$. Clearly this function $g$ satisfies all the conditions of the lemma.

(iii) We have now only to consider the case where $\mathscr{2}^{+}$is empty. If $\mathscr{Q}^{-}$is also empty, we may take $g=i f$. As in case (ii) (b), we may assume that, if $\mathscr{2}^{-}$is not empty, then there is a function $q_{-}$such that $q_{-} / q$ is ultimately monotonic increasing for every $q$ in $\mathscr{Q}^{-}$other than $q_{-}$. It now clearly suffices to take

$$
g(x)=i(x) q_{-}(x) \sup _{\mathbf{0} \leqq \xi \leqq x}\left\{f(\xi) / q_{-}(\xi)\right\},
$$

for all $x \geqq 0$. This completes the proof.

COROLlary. Suppose that $f$ and $\mathscr{Q}$ satisfy the conditions of lemma 5 . Then there exists a function $g$ in $\mathscr{C}$ which is monotonically comparable with each element cf $\mathscr{Q}$ and satisfies $t>g>q$ for every $q$ in $\mathscr{Q}$ with $q \prec t$.

Proof. Let $f^{\prime}=k / f$ and $q^{\prime}=k / q$ for each $q \in \mathscr{Q}$ and apply lemma 5 to $f^{\prime}$ and the set $\mathscr{Q}^{\prime}$ of all the functions $q^{\prime}$.

Lemma 6. Suppose $t, u, f \in \mathscr{C}$ and are such that $t \prec f \prec u$, and $t, u$ are monotonically comparable: then there exists an $s$ in $\mathscr{C}$ which is monotonically comparable with $t$ and $u$, and which satisfies neither $f \prec s$, nor $f \succ s$.

Proof. We define a sequence of real numbers $\left\{\beta_{r}\right\}$ and a sequence of functions $\left\{s_{r}\right\}$ by the following inductive process: 
(i) $\beta_{1}=0, s_{1}(x)=u(x)$ for $x \geqq 0$;

(ii) if $\beta_{1}, \beta_{2}, \cdots, \beta_{2 r-1} ; s_{1}, s_{2}, \cdots, s_{2 r-1}$ have been defined we first choose $\beta_{2 r}$ so that

$$
\beta_{2 r}>\beta_{2 r-1}+1
$$

and

$$
s_{2 r-1}\left(\beta_{2 r}\right)>f\left(\beta_{2 r}\right) ;
$$

then define $s_{2 r}(x)$ by

$$
\left\{\begin{array}{l}
s_{2 r}(x)=s_{2 r-1}(x), \quad \text { if } 0 \leqq x \leqq \beta_{2 r}, \\
s_{2 r}(x)=\frac{s_{2 r-1}\left(\beta_{2 r}\right)}{t\left(\beta_{2 r}\right)} t(x)_{2 r-1} \text { if } \beta_{2 r} \leqq x ;
\end{array}\right.
$$

(iii) If $\beta_{1}, \beta_{2}, \cdots, \beta_{2 r} ; s_{1}, s_{2}, \cdots, s_{2 r}$ have been defined, we first choose $\beta_{2 r+1}$ greater than $\beta_{2 r}$ and such that

$$
s_{2 r}\left(\beta_{2 r+1}\right) \leqq f\left(\beta_{2 r+1}\right),
$$

then define $s_{2 r+1}(x)$ by

$$
\left\{\begin{array}{l}
s_{2 r+1}(x)=s_{2 r}(x), \text { if } 0<x \leqq \beta_{2 r+1}, \\
s_{2 r+1}(x)=\frac{s_{2 r}\left(\beta_{2 r+1}\right)}{u\left(\beta_{2 r+1}\right)} u(x), \text { if } \beta_{2 r+1} \leqq x .
\end{array}\right.
$$

Finally, put $s(x)=\lim _{r \rightarrow \infty} s_{r}(x)$. Note that (9) is again true. It follows that $s(x)$ is continuous for all $x$, and (15), (17) together imply that, for each integer $r$, there exists $\xi_{r}$ satisfying $\beta_{r}<\xi_{r}<\beta_{r+1}$ and $s\left(\xi_{r}\right)=s_{r+1}\left(\xi_{r}\right)=$ $f\left(\xi_{r}\right)$. By (14), $\beta_{r} \rightarrow \infty$ as $r \rightarrow \infty$ and therefore neither $f \succ s$, nor $f \prec s$ can be true. The conditions (16) and (18) imply that $s$ is monotonically comparable with $t$ and $u$.

REMARK. It is clear that the function $s$ defined above must satisfy $t \prec s \prec u$.

We now prove a result which seems to be of some interest in itself.

THEOREM 2. If $\mathscr{T}$ is a countable set of functions of $\mathscr{C}$ any two of which are monotonically comparable, and if $f$ is in $\mathscr{C}$ and is comparable with each element of $\mathscr{T}$, then there exists a function $g$ of $\mathscr{C}$, which is monotonically comparable with each element of $\mathscr{T}$, but which satisfies neither $g\langle f$ nor $g \succ f$.

Proof. We may clearly suppose that no function of $\mathscr{T}$ is equivalent to $t$. Let $\mathscr{Q}, \mathscr{R}$ be the subsets of $\mathscr{T}$ such that $t\langle t, t\rangle t$ respectively for $t$ in $\mathscr{Q}, \mathscr{R}$. Suppose first that at least one of $\mathscr{Q}, \mathscr{R}$ is empty, say $\mathscr{R}$. Then $t \prec f$ for all $t \epsilon T$. Let $u$ in $\mathscr{C}$ be such that $u$ is monotonically comparable with $t$ and $t \prec u \prec f$ for all $t \in \mathscr{T}$ ( $u$ exists by lemma 5 ). Put

$$
g(x)=u(x) \sup _{0 \leqq \xi \leqq x} \frac{f(\xi)}{u(\xi)}, \text { for all } x>0 .
$$


Then $g(x) \geqq f(x)$ for all $x$ and there is a sequence $\left\{\xi_{r}\right\}$ of real numbers such that $\xi_{n} \rightarrow \infty$ as $n \rightarrow \infty$ and $g\left(\xi_{n}\right)=f\left(\xi_{n}\right)$. Since $g(x) / u(x)$ is monotonic for all $x$, it follows that $g$ satisfies the requirements of the theorem.

On the other hand, suppose that neither of $\mathscr{Q}, \mathscr{R}$ are empty. By lemma 5 we can find functions $u, v$ which are monotonically comparable with each element of $\mathscr{T}$, satisfy $u \prec t \prec v$ and are such that $t\langle u, t\rangle v$ respectively for elements $t$ in $\mathscr{Q}, \mathscr{R}$. Now apply lemma 6 to obtain a function $g$ which is monotonically comparable with $u, v$ and satisfies neither $g \succ f$ nor $g \prec f$. This function $g$ is clearly monotonically comparable with each element of $\mathscr{T}$. This completes the proof of the theorem.

The result of theorem 2 allows us to strengthen lemmas 1 and 2 , to the case where the functions are monotonically comparable.

LEMMA 7. If $\mathscr{T}$ is a countable set of functions of $\mathscr{C}$, any two of which are monotonically comparable, there is a function $u$ in $\mathscr{C}$ which is monotonically comparable with each element of $\mathscr{T}$ and satisfies $t \prec u$ for each $t$ in $\mathscr{T}$.

LEMMA 8. If $\mathscr{T}$ is a countable set of functions of $\mathscr{C}$, any two of which are monotonically comparable, and $t_{0}$ in $\mathscr{T}$ is such that $t_{0} \prec t$ for each $t$ in $\mathscr{T}$ other than $t_{0}$, there is a function $u$ in $\mathscr{C}$ which is monotonically comparable with each function of $\mathscr{T}$ and satisfies $t_{0} \prec u<t$ for each $t$ in $\mathscr{T}$ other than $t_{0}$.

Proof of Lemma 7. If $\mathscr{T}$ contains a maximal element $m$, such that, for every $t \in \mathscr{T}, t \leq m$, then we use the argument of lemma 5 to adjoin a new element $m_{0}$ to $\mathscr{T}$, such that $m_{0}$ is equivalent to all such elements $m$, and $m_{\mathbf{0}} / t$ is ultimately monotonic increasing for every $t$ in $\mathscr{T}$ other than $m_{0}$. It then suffices to take $u(x)=i(x) m_{0}(x)$.

On the other hand, if $\mathscr{T}$ has no maximal element, then there exists a sequence $t_{1} \prec t_{2} \prec \cdots \prec t_{n} \prec \cdots$ of functions of $\mathscr{T}$ such that, for each $t \in \mathscr{T}, t \prec t_{r}$ for sufficiently large integers $r$. Apply lemma 1 to obtain a function $f$ in $\mathscr{C}$ such that $f>t_{n}$ for each integer $n$. Then $f>t$ for each $t \in \mathscr{T}$. Apply theorem 2 to obtain a function $u$ which is monotonically comparable with each $t \in \mathscr{T}$ and satisfies neither $u \prec t$ nor $u \succ t$. It is clear that $u>t$ for each $t \in \mathscr{T}$.

Proof of Lemma 8. This is just a special case of lemma 5 for which $f=t_{0}$. Our next object is to extend Theorem 2 and lemmas 7,8 to the case where $\mathscr{T}$ need not be enumerable, but has an enumerable basis. Suppose $\mathscr{T} \subset \mathscr{C}$ is any set of functions and $\mathscr{B} \subset \mathscr{T}$ : then we say that $\mathscr{B}$ forms a basis for $\mathscr{T}$ if every element in $\mathscr{T}$ can be written as a finite product of real powers of elements in $\mathscr{B}$. The set $\mathscr{T}$ has a countable basis if there is some countable $\mathscr{B}$ which forms a basis for $\mathscr{T}$. We need two additional lemmas.

Leмma 9. If $\mathscr{T}$ is an irreducible scale which has a countable basis, there is a sequence $t_{1}, t_{2}, \cdots, t_{n}, \cdots$ of elements $\mathscr{T}$ such that $t_{n} \succ k$ and, for any $t$ in $\mathscr{T}$ with $t \succ k$, there are integers $r, s$ with $k \prec t_{r} \prec t \prec t_{s}$. 
Proof. Let $e_{1}, e_{2}, \cdots, e_{n}, \cdots$ be a basis for $\mathscr{T}$ chosen so that $e_{r}>k$ for each integer $r$. We suppose, without any loss of generality, that the basis is such that its elements are independent in the sense that

$$
e_{1}^{\alpha_{1}} e_{2}^{\alpha_{2}} \cdots e_{r}^{\alpha_{r}}(x) \rightarrow \beta, \text { as } x \rightarrow+\infty, 0<\beta<+\infty,
$$

holds only when $\alpha_{1}=\alpha_{2}=\cdots=\alpha_{r}=0$.

Let $\mathscr{T}_{r}$ be the set of functions which can be written in the form

$$
t=e_{1}^{\alpha_{1}} e_{2}^{\alpha_{2}} \cdots e_{r}^{\alpha_{r}}
$$

for some real numbers $\alpha_{1}, \alpha_{2}, \cdots, \alpha_{r}$. Since the elements $e_{1}, e_{2}, \cdots, e_{r}$ are independent, there is a $(1,1)$ correspondence between the points $\left(\alpha_{1}, \alpha_{2}, \cdots\right.$, $\alpha_{r}$ ) of Euclidean $r$-space and the functions

$$
t=e\left(\alpha_{1}, \alpha_{2}, \cdots, \alpha_{r}\right)=e_{1}^{\alpha_{1}} e_{2}^{\alpha_{2}} \cdots e_{r}^{\alpha_{r}}
$$

of $\mathscr{T}_{r}$.

Since $\mathscr{T}$ is completely ordered by the relation $\prec$, the correspondence induces a total ordering in $r$-dimensional space. Let this ordering be denoted by the same symbol and let $E_{-}$be the set of points $\left(\alpha_{1}, \alpha_{2}, \cdots, \alpha_{r}\right)$ such that

$$
\left(\alpha_{1}, \alpha_{2}, \cdots, \alpha_{r}\right) \prec(0,0, \cdots, 0) ;
$$

$E_{+}$be the set of points $\left(\alpha_{1}, \alpha_{2}, \cdots, \alpha_{r}\right)$ such that

$$
\left(\alpha_{1}, \alpha_{2}, \cdots, \alpha_{r}\right)>(0,0, \cdots, 0) \text {. }
$$

$E_{-} \cup E_{+}$forms the whole of $r$-dimensional space apart from $(0,0, \cdots, 0)$. It is easy to verify that $E_{-}, E_{+}$must be convex sets in $r$-space. It follows, without difficulty, that $E_{+}$can be represented as a union $E_{+}=\cup_{i=1}^{r} H_{i}$, where $H_{i}$ is an open $i$-dimensional half space, lying in the bounding hyperplane of $H_{i+1}$ when $i<r$, and bounded by an $(r-1)$-dimensional hyperplane passing through the origin.

Now take an enumerable set of points $\left(\alpha_{1}^{(n)}, \alpha_{2}^{(n)}, \cdots, \alpha_{r}^{(n)}\right)$ which is dense in both $H_{1}$ and $H_{r}$ and put

$$
t_{r}^{(n)}=e\left(\alpha_{1}^{(n)}, \alpha_{2}^{(n)}, \cdots, \alpha_{r}^{(n)}\right), \quad n=1,2, \cdots .
$$

If $t \in \mathscr{T}_{r}$ and $t \succ k$, there will be integers $m, p$, for which

$$
k \prec t_{r}^{(m)} \prec t<t_{r}^{(p)} \text {. }
$$

If we now reorder the elements $t_{r}^{(n)}, n=1,2, \cdots, r=1,2, \cdots$ in a single sequence $t_{1}, t_{2}, \ldots$ of elements of $\mathscr{T}$, we have satisfied the conditions of the lemma since $\mathscr{T}=\cup_{r=1}^{\infty} \mathscr{T}_{r}$.

Lemma 10. If $\mathscr{S}$ is an irreducible scale which has a countable basis, and $f$ in $\mathscr{C}$ is comparable with each element of $\mathscr{S}$, then there is a sequence $s_{1}, s_{2}, \cdots$, $s_{r}, \cdots$ of elements of $\mathscr{S}$ such that (i), if $s \in \mathscr{S}$ and $s>t$, there is an integer $r$ such that $s>s_{r}>f$, and (ii), if $s \in \mathscr{S}$ and $s<f$, there is an integer $p$ such that $s \prec s_{p} \prec t$. 
Proof. If $f$ is equivalent to an element $f^{\prime}$ in $\mathscr{S}$, the result follows immediately on applying lemma 9 to the function $s / f^{\prime}$ or $f^{\prime} / s$ in cases (i) and (ii). Therefore, we may assume that $f$ is not equivalent to any element in $\mathscr{S}$.

We now modify the method of proof used in lemma 9 . Let $e_{1}, e_{2}, \cdots$ be an independent basis for the set $\mathscr{S}$. Let $\mathscr{S}_{r}$ be the set of elements of $\mathscr{S}$ which can be represented in the form

$$
s=e_{1}^{\alpha_{1}} e_{2}^{\alpha_{2}} \cdots e_{r}^{\alpha_{r}},
$$

where $\alpha_{1}, \alpha_{2}, \cdots, \alpha_{r}$ are real numbers. We divide the points $\left(\alpha_{1}, \alpha_{2}, \cdots, \alpha_{r}\right)$ of $r$-dimensional space into two sets $E^{-}$and $E^{+}$, putting $\left(\alpha_{1}, \alpha_{2}, \cdots, \alpha_{r}\right)$ in $E^{-}$, if $e\left(\alpha_{1}, \alpha_{2}, \cdots, \alpha_{r}\right) \prec f$, and in $E^{+}$, if $e\left(\alpha_{1}, \alpha_{2}, \cdots, \alpha_{r}\right) \succ f$.

The two sets $E^{-}$and $E^{+}$constitute a convex partition of space into two disjoint sets. If one of these sets, say $E^{+}$, is empty, it is sufficient to take for $s_{r}^{(1)}, s_{r}^{(2)}, \cdots, s_{r}^{(n)}, \cdots$ any set of functions corresponding to a dense set of points $\left(\alpha_{1}^{(n)}, \alpha_{2}^{(n)}, \cdots, \alpha_{r}^{(n)}\right) n=1,2, \cdots$, in $r$-dimensional space. If both the sets $E^{-}, E^{+}$are non-empty, we proceed as follows. Let $H_{r-1}$ be the $(r-1)$ dimensional hyperplane separating $E^{-}$and $E^{+}$. Let $E_{r-1}^{-}=E^{-} \cap H_{r-1}$, $E_{r-1}^{+}=E^{+} \cap H_{r-1}$. If each of these sets is non-empty, let $H_{r-2}$ be the $(r-2)$ dimensional hyperplane separating $E_{r-1}^{-}, E_{r-1}^{+}$; and put

$$
E_{r-2}^{-}=E^{-} \cap H_{r-2}, \quad E_{r-2}^{+}=E^{+} \cap H_{r-2} \text {. }
$$

If each of the sets $E_{r-m}^{-}, E_{r-m}^{+}(m \geqq 2)$ is not empty, let $H_{r-m-1}$ be the $(r-m-1)$-dimensional hyperplane separating them, and put

$$
E_{r-m-1}^{-}=E^{-} \cap H_{r-m-1}, \quad E_{r-m-1}^{+}=E^{+} \cap H_{r-m-1} .
$$

In this way, we obtain inductively sets

$$
\begin{aligned}
& E_{p}^{-} \subset E_{p+1}^{-} \subset \cdots \subset E_{r-1}^{-} \subset E^{-} \\
& E_{p}^{+} \subset E_{p+1}^{+} \subset \cdots \subset E_{r-1}^{+} \subset E^{+}
\end{aligned}
$$

where $p \geqq 0$ is an integer, one of the sets $E_{p}^{-}, E_{p}^{+}$is empty and none of the sets $E_{p+1}^{-}, \cdots, E_{r-1}^{-}, E_{p+1}^{+}, \cdots E_{r-1}^{+}$is empty. Now let $\left(\alpha_{1}^{(n)}, \alpha_{2}^{(n)}, \cdots, \alpha_{r}^{(n)}\right)$ $n=1,2, \cdots$ be a set of points in $r$-space which is dense in each of the sets (19); and put $s_{r}^{(n)}=e\left(\alpha_{1}^{(n)}, \alpha_{2}^{(n)}, \cdots, \alpha_{r}^{(n)}\right)$. It is easy to see that, if $s>t$ and $s \in \mathscr{S}_{r}$, then there is an integer $m$ for which $s>s_{r}^{(m)} \succ f$, while, if $s<f$ and $s \in \mathscr{S}_{r}$, then there is an integer $p$ for which $s<s_{r}^{(p)} \prec t$.

Finally, rearrange the functions $s_{r}^{(n)}, n=1,2, \cdots, r=1,2, \cdots$ as a single sequence $s_{1}, s_{2}, \cdots$, and it follows that this sequence has the desired properties.

Remark. The method of proof used in lemmas 9 and 10 can be extended to prove the following more general result:

TheOREM. If $\mathscr{S}$ is an irreducible scale which has a countable basis, and $\mathscr{T}$ is any subset of $\mathscr{S}$, then there is a sequence $t_{1}, t_{2}, \cdots, t_{r}, \cdots$ of elements of $\mathscr{T}$ 
such that, for every $t \in \mathscr{T}$, there are integers $r$ and $s$ such that

$$
t_{r} \leq t \leq t_{s}
$$

We omit the proof since this theorem is not needed in the sequel.

The next theorem is an extension of lemmas 1 and 2 .

THEOREM 3. If $\mathscr{S}$ is an irreducible scale with a countable basis, there are functions $f, g$ which are comparable with each element of $\mathscr{S}$ and satisfy

$$
g>s>1 / g \text { for every } s \in \mathscr{S},
$$

if $s \in \mathscr{S}$ and $s>k$, then $s>t>k$,

if $s \in \mathscr{S}$ and $s<k$, then $s<1 / t<k$.

If, in addition, any two elements of $\mathscr{S}$ are monotonically comparable, then $f, g$ can be chosen to be monotonically comparable with each element of $\mathscr{S}$.

Proof. Let $s_{1}, s_{2}, \cdots$ be a sequence of elements of $\mathscr{P}$ satisfying the conditions of lemma 9. Apply lemma 1 to obtain a function $g$ satisfying

$$
g>s_{n} \text { for each integer } n \text {, }
$$

and apply lemma 2 to obtain a function $f$ satisfying

$$
s_{n} \succ f>k \text { for each integer } n \text {. }
$$

It follows immediately that the functions $f, g$ have the required properties.

If $\mathscr{S}$ is such that its elements are monotonically comparable, the result follows by using lemmas 7 and 8 instead of 1 and 2 .

REMARK. This theorem shows clearly that a scale which has a countable basis cannot have any of the properties $\left(\mathrm{P}_{5}\right),\left(\mathrm{P}_{6}\right)$ of $\left(\mathrm{P}_{7}\right)$.

The next theorem is an extension of theorem 2 and will be important in the sequel.

THEOREM 4. If $\mathscr{S}$ is an irreducible scale, any two of whose elements are monotonically comparable, $\mathscr{S}$ has a countable basis, and $f \in \mathscr{C}$ is comparable with each element of $\mathscr{S}$; then there is an element $g \in \mathscr{C}$ which is monotonically comparable with each element of $\mathscr{S}$, but which satisfies neither $g \succ f$ nor $g \prec f$.

Proof. Apply lemma 10 to obtain a sequence of functions $s_{1}, s_{2}, \ldots, s_{n}, \ldots$ in $\mathscr{S}$ with the properties stated. Let this sequence form the countable set $\mathscr{T}$, and apply theorem 2 to obtain a function $g \in \mathscr{C}$ which is monotonically comparable with each element of $\mathscr{T}$ but satisfies neither $g \succ f$ nor $g \prec f$. If $s$ is any element of $\mathscr{S}$ and $s \succ t$, it follows that there is an integer $n$ such that $s \succ s_{n} \succ f$. Since $s_{n}$ is comparable with $g$, we must have $s \succ s_{n} \succ g$, and therefore $g$ is monotonically comparable with $s$. Similarly, when $s<f, g$ is also monotonically comparable with $s$. This completes the proof of the theorem. 


\section{The existence of a maximal dense scale}

In this section and the following, we have been forced to make use of the continuum hypothesis. Since a continuous function is determined by the values it takes on the rationals, the set $\mathscr{C}$ has the power $2^{N_{0}}$ of the continuum. We suppose the subset $\mathscr{C}^{\infty}$ of the functions of $\mathscr{C}$, which tend to $+\infty$ as $x$ tends to $+\infty$, is well-ordered in a transfinite sequence

$$
f_{1}, f_{2}, \cdots, f_{\mu}, \cdots, \quad \mu<\Omega,
$$

where $\Omega$ is the first ordinal of power greater than $\boldsymbol{\aleph}_{0}$. Then to each ordinal $\mu$ with cardinal less than $2^{\aleph_{0}}$, there will be a corresponding function $f_{\mu} \in \mathscr{C}^{\infty}$; and the functions $f_{\mu}$ exhaust $\mathscr{C}^{\infty}$, as $\mu$ runs through each such ordinal. We suppose, as we may, that the well-ordering is chosen so that $f_{1}=i$. We give an inductive definition for a scale $\mathscr{S}_{\mu}$ for each ordinal $\mu<\Omega$ and finally take $\mathscr{S}=\cup_{\mu<\Omega} \mathscr{S}_{\mu}$.

First let $\mathscr{S}_{1}$ be the scale of functions $i^{\alpha}$ for all real $\alpha$. It is clear that $\mathscr{S}_{1}$ has the properties $\left(\mathrm{P}_{1}^{*}\right),\left(\mathrm{P}_{2}\right)-\left(\mathrm{P}_{4}\right)$, and $\mathscr{S}_{1}$ has a countable basis.

Now suppose that $\tau<\Omega$, and that scales $\mathscr{S}_{\mu}$ have been defined for $\mu<\tau$ with the following properties:

(20) for $\mu<\tau, \mathscr{S}_{\mu}$ has properties $\left(\mathrm{P}_{1}^{*}\right),\left(\mathrm{P}_{2}\right)-\left(\mathrm{P}_{4}\right)$;

(21) for $\mu<\tau, \mathscr{S}_{\mu}$ has a countable basis;

(22) for $\mu<\tau$, there are functions $s, t$ in $\mathscr{S}_{\mu}$ such that $k \prec s \prec t_{\mu} \prec t$;

(23) for $\mu<\tau$, there is a function $g \in \mathscr{S}_{\mu}$ such that neither $g \succ f_{\mu}$ nor $g \prec f_{\mu}$;$$
\text { for } 1 \leqq \mu<\nu<\tau, \mathscr{S}_{\mu} \subset \mathscr{S}_{\nu} \text {. }
$$

The conditions (20)-(24) are clearly satisfied when $\tau=2$. Our object is to show that $\mathscr{S}_{\tau}$ can be defined so that the conditions are satisfied with $\tau$ replaced by $(\tau+1)$.

Let

$$
\mathscr{T}_{\tau}=\underset{\mu<\tau}{\cup} \mathscr{S}_{\mu}, \quad 2 \leqq \tau<\Omega .
$$

By the continuum hypothesis, $\mathscr{T}_{\tau}$ is an enumerable union of scales each with a countable basis, and so $\mathscr{T}_{\tau}$ itself has a countable basis.

If there is an element $t$ in $\mathscr{T}_{\tau}$ such that $t>t_{\tau}$, we put $\mathscr{T}_{\tau}^{\prime}=\mathscr{T}_{\tau}$; otherwise we aim to form a scale $\mathscr{T}_{\tau}^{\prime}$, from $\mathscr{T}_{\tau}$, by adjoining a suitable element to $\mathscr{T}_{\tau}$. By theorem 3, there exists a function $g$, which is monotonically comparable with each element of $\mathscr{T}_{\tau}$ and satisfies $g>t$ for every $t \epsilon \mathscr{T}_{\tau}$. By lemma 4 (with $f=k$ ), there is a function $u$ which is monotonically comparable with $g$ (and therefore with each element of $\mathscr{T}_{T}$ ) and satisfies

$$
u>g, \quad u>f_{\tau} \text {. }
$$


It follows that the set of functions of the form $u^{\alpha} t$, where $\alpha$ is real and $t \in \mathscr{T}_{\tau}$, is a scale, with properties $\left(\mathrm{P}_{1}^{*}\right),\left(\mathrm{P}_{2}\right)-\left(\mathrm{P}_{4}\right)$, which has a countable basis. We define $\mathscr{T}_{\tau}^{\prime}$ to be this new scale.

If there is an element $s$ in $\mathscr{T}_{\tau}^{\prime}$ such that

$$
k \prec s \prec t_{\tau} \text {, }
$$

we put $\mathscr{T}_{\tau}^{\prime \prime}=\mathscr{T}_{\tau}^{\prime}$; otherwise we aim to form $\mathscr{T}_{\tau}^{\prime \prime}$, from $\mathscr{T}_{\tau}^{\prime}$, by adjoining such an element to it. By applying theorem 3 to $\mathscr{T}_{\tau}^{\prime}$, we can find a function $h \in \mathscr{C}$, which is monotonically comparable to each element of $\mathscr{T}_{\tau}^{\prime}$, and satisfies

$$
k \prec h \prec t \text { for } t \text { in } \mathscr{T}_{\tau}^{\prime} \cap \mathscr{C}^{\infty} \text {. }
$$

By lemma 4, there is a function $l$ which is monotonically comparable with $k$ and $h$ (and therefore with each element of $\mathscr{T}_{\tau}^{\prime}$ ) and satisfies $k \prec l \prec h$ and $l \prec f_{\tau}$. We now define $\mathscr{T}_{\tau}^{\prime \prime}$ to be the set of functions of the form $l^{\alpha} t$, where $\alpha$ is real, and $t \in \mathscr{T}_{\tau}^{\prime}$. Then $\mathscr{T}_{\tau}^{\prime \prime}$ is a scale with the properties $\left(\mathrm{P}_{1}^{*}\right),\left(\mathrm{P}_{2}\right)-\left(\mathrm{P}_{\mathbf{4}}\right)$ and has a countable basis.

Now, it may happen that there is an element $g \in \mathscr{T}_{r}^{\prime \prime}$, such that neither $g \succ f_{\tau}$ nor $g \prec f_{\tau}$. In this case we put $\mathscr{T}_{\tau}^{\prime \prime \prime}=\mathscr{T}_{\tau}^{\prime \prime} ;$ otherwise we again form a new scale by adjoining a suitable element to $\mathscr{T}_{r}^{\prime \prime}$. Clearly $f_{\tau}$ must be comparable with each element of $\mathscr{T}_{\tau}^{\prime \prime}$, so we can apply theorem 4 to obtain the required $g$. Finally, we let $\mathscr{T}_{\tau}^{\prime \prime \prime}$ be the set of all functions of the form $g^{\alpha} t$ where $\alpha$ is real and $t \epsilon \mathscr{T}_{\tau}^{\prime \prime}$.

Define $\mathscr{S}_{\tau}=\mathscr{T}_{\tau}^{\prime \prime \prime}$. If $\tau$ is replaced by $(\tau+1)$, it is clear that each of conditions (20)-(23) is satisfied. The condition (24) is immediate since $\mathscr{S}_{\tau}=$ $\mathscr{T}_{\tau}^{\prime \prime \prime} \supset \mathscr{T}_{\tau}^{\prime \prime} \supset \mathscr{T}_{\tau}^{\prime} \supset \mathscr{T}_{\tau} \supset \mathscr{S}_{\mu}$ for all $\mu<\tau$.

By the wording of the conditions $(20)-(24)$, it makes no difference to the construction of $\mathscr{S}_{\tau}$ whether $\tau$ has an immediate predecessor or is a limit ordinal. Thus, by induction, $\mathscr{S}_{\mu}$ is defined for all $\mu<\Omega$.

If $\mathscr{S}=\cup_{\mu<\Omega} \mathscr{S}_{\mu}$, it is immediate that $\mathscr{S}$ has the properties $\left(\mathrm{P}_{1}^{*}\right)$, $\left(\mathrm{P}_{2}\right)-\left(\mathrm{P}_{4}\right)$; but, naturally, $\mathscr{S}$ will not have a countable basis. We now show that $\mathscr{S}$ also has the properties $\left(\mathrm{P}_{5}\right)-\left(\mathrm{P}_{7}\right)$. If $f \in \mathscr{C}$, then $f^{\prime}=\max (f, i) \in \mathscr{C}^{\infty}$ and therefore $f^{\prime}=f_{\mu}$, for some ordinal $\mu<\Omega$. Hence there is an element $s \in \mathscr{S}_{\mu}$, such that $s>f^{\prime}$ and therefore $s>t$. Since $s \in \mathscr{S}$, this proves that $\mathscr{S}$ has property $\left(\mathrm{P}_{6}\right)$. Now suppose $g \in \mathscr{C}^{\infty}$; then $g=f_{\sigma}$, for some ordinal $\sigma<\Omega$. There exists $s$ in $\mathscr{S}_{\sigma}$ with $k \prec s \prec g$. Since $s \in \mathscr{S}$, this proves that $\mathscr{S}$ has property $\left(\mathrm{P}_{7}\right)$.

Finally, suppose $f$ in $\mathscr{C}$ is comparable with each element of $\mathscr{S}$. Then, since $k \in \mathscr{S}$, either $f$ is equivalent to $k$, or $f \in \mathscr{C}^{\infty}$ or $f \in \mathscr{C}^{0}$. Thus, there is an ordinal $v<\Omega$ such that either $f=f_{\nu}$ or $1 / f=f_{\nu}$. There is an element $s \in \mathscr{S}_{\nu}$ such that neither $s \succ f_{\nu}$ nor $s \prec f_{\nu}$. Hence, either $s$ or $1 / s$, both of which are elements of $\mathscr{S}$, is equivalent to $f$. This proves that the scale $\mathscr{S}$ has the property $\left(\mathrm{P}_{5}\right)$; i.e. it is maximal.

Thus we have proved 
THEOREM 5. (Assuming the continum hypothesis). There is an irreducible scale $\mathscr{S}$ which is maximal and dense and for which any two elements are monotonically comparable.

REMARK. It is perhaps worth asking whether anything like theorem 5 can be proved without using the continuum hypothesis. In fact the same methods work, if we redefine properties $\left(\mathrm{P}_{5}\right),\left(\mathrm{P}_{6}\right),\left(\mathrm{P}_{7}\right)$ relative to a subset $\mathscr{A} \subset \mathscr{C}$, for which we know that the power of $\mathscr{A}$ is $\boldsymbol{N}_{1}$, where $\boldsymbol{N}_{1}$ is the smallest cardinal greater than $\boldsymbol{\aleph}_{0}$. Thus we say that a scale $\mathscr{S}$ is maximal relative to $\mathscr{A}$ if it has property: $\left(\mathrm{P}_{5 \mathscr{A}}\right)$ if $f \in \mathscr{A}$ and is comparable with each element of $\mathscr{S}$, then $f$ is equivalent to some element of $\mathscr{S}$.

We say that a scale $\mathscr{S}$ is dense in $\mathscr{A}$, if it has properties $\left(\mathrm{P}_{\mathbf{6}_{\mathscr{A}}}\right)$ and $\left(\mathrm{P}_{7 \mathscr{A}}\right)$ obtained by making similar modifications to $\left(\mathrm{P}_{6}\right)$ and $\left(\mathrm{P}_{7}\right)$. The method used to prove theorem 5 then gives, without using the continuum hypothesis:

THEOREM $5 \mathscr{A}$. Let $\mathscr{A}$ be any subset of $\mathscr{C}$ of cardinal $\boldsymbol{\aleph}_{1}$. Then there is an irreducible scale $\mathscr{S}$ which is maximal relative to $\mathscr{A}$, and dense in $\mathscr{A}$, and for which any two elements are monotonically comparable.

\section{Existence of a scale which is maximal but not dense}

In this section, we again need to use the continuum hypothesis, but we modify the method of construction of theorem 5 , so that the scale $\mathscr{S}$ obtained has neither of the properties $\left(\mathrm{P}_{6}\right)$ or $\left(\mathrm{P}_{7}\right)$, though the other properties are preserved. The idea behind our construction is to ensure, (i) that every function in $\mathscr{S}$ has, for some arbitrarily large values of $x$, values which are not too large; (ii) that every function in $\mathscr{S}_{\cap} \mathscr{C}^{\infty}$ has, for some arbitrarily large values of $x$, values which are not too small.

Thus we say that two functions $f, g$ in $\mathscr{C}$ are exponentially similar, if they are monotonically comparable and, in addition

$$
\lim _{x \rightarrow+\infty} \inf \left\{x^{-1}|\log f(x)-\log g(x)|\right\}=0 .
$$

A subset $\mathscr{E} \subset \mathscr{C}$ is said to have the property $\left(\mathrm{P}_{8}\right)$ if any two of its elements are exponentially similar. We say that two functions $f, g \in \mathscr{C}$ are logarithmically different, if they are monotonically comparable and, in addition

$$
\lim _{x \rightarrow+\infty} \sup \left|\frac{\log f(x)-\log g(x)}{\log \log x}\right|=+\infty .
$$

A subset $\mathscr{E} \subset \mathscr{C}$ is said to have the property $\left(\mathrm{P}_{9}\right)$ if any two of its elements, which are not equivalent, are logarithmically different. A subset $\mathscr{E} \subset \mathscr{C}$ is said to be a restricted set of functions if it has both the properties $\left(\mathrm{P}_{8}\right)$ and $\left(\mathrm{P}_{9}\right)$.

Our object is to obtain a scale $\mathscr{S}$ which has the properties $\left(\mathrm{P}_{1}^{*}\right),\left(\mathrm{P}_{2}\right)-\left(\mathrm{P}_{5}\right)$ and $\left(\mathrm{P}_{8}\right),\left(\mathrm{P}_{9}\right)$. Such a scale will have neither of the properties $\left(\mathrm{P}_{6}\right),\left(\mathrm{P}_{7}\right)$. 
In the first place take $f(x)=\exp x$. Then no function $f$ of $\mathscr{P}$ which is exponentially similar to the function $k$ of $\mathscr{S}$ can satisfy $g \succ t$. Hence $\mathscr{S}$ has not got property $\left(\mathrm{P}_{6}\right)$. Secondly, take

$$
\begin{array}{ll}
f(x)=1, & \text { for } 0 \leqq x \leqq e, \\
f(x)=\log x, & \text { for } e \leqq x .
\end{array}
$$

Then $f \succ k$, but there is no function $g$ in $\mathscr{C}$ which is logarithmically different from $k$, and satisfies $f \succ g \succ k$. Hence $\mathscr{S}$ has not got property $\left(\mathrm{P}_{7}\right)$.

To obtain a scale $\mathscr{S}$ which is maximal, and at the same time a restricted set, it is necessary to modify some of the methods and results of section 4 . We will state the results and sketch the arguments at those points where care is needed.

In section 4, we made repeated use of the fact that, if $f, g, h$ are in $\mathscr{C}$ and $f \prec g \prec h$, and, if $f$ and $g$ are monotonically comparable and $g$ and $h$ are monotonically comparable, then $f$ and $h$ are monotonically comparable. The new situation is rather more complicated; the most useful results are summarised in the following lemma.

LEMMA 11. Let $f, g, h$ be monotonically comparable functions of $\mathscr{C}$ satisfying

$$
f<g<h \text {. }
$$

If $f$ and $h$ are exponentially similar, then so are $f$ and $g$, and $g$ and $h$. If $f$ and $g$ or $g$ and $h$ are logarithmically different then so are $f$ and $h$.

Lemma 5 obviously has to be weakened slightly in its new form. It remains strong enough for our purposes.

Lemma 5A. Suppose $\mathscr{Q}$ is countable and forms a restricted set of functions of $\mathscr{C}$, and $f$ is comparable with every function in $\mathscr{Q}$, and there is at least one $q$ of $\mathscr{Q}$ with $q \succ f$. Then either (a) there exists a function $q_{+}$of $\mathscr{Q}$ such that $f \prec q_{+} \prec q$, for every $q$ in 2 , other than $q_{+}$, which satisfies $q>f$; or (b) there exists a function $g \in \mathscr{C}$ such that the set $\mathscr{Q} \cup\{g\}$ is a restricted set and $f \prec g \prec q$, for every $q$ in $\mathscr{Q}$ satisfying $q>f$.

Proof. Note first that, since any two elements of $\mathscr{2}$ are logarithmically different, no two elements of $\mathscr{Q}$ can be equivalent. Hence, if (i) is not satisfied, we must be in the situation of case (i) of lemma 5 . Thus it is sufficient to prove that (b) is satisfied when the set $2^{+}$of elements $q>t$ has no minimal element. We modify the proof of (i) of lemma 5 as follows. Previously, we chose a sequence $\left\{\alpha_{r}\right\}$ of real constants inductively so that, when $\alpha_{1}, \alpha_{2}, \cdots, \alpha_{r}$ were known, $\alpha_{r+1}$ was chosen large enough to satisfy (4)-(7). We now choose $\alpha_{r+1}$ so that in addition it satisfies

$$
\inf _{\alpha_{r} \leqq x \leqq \alpha_{r+1}} x^{-1}\left|\log s_{r}(x)-\log q_{s}(x)\right|<\frac{1}{r}, \quad s=1,2, \cdots, r ;
$$

and 


$$
\sup _{\alpha_{r} \leqq x \leqq \alpha_{r+1}} \frac{\left|\log s_{r}(x)-\log q_{s}(x)\right|}{\log \log x}>r, \quad s=1,2, \cdots, r \text {, }
$$

unless $q_{s}=v_{r}$.

The conditions (25), (26) are sufficient to ensure that, when $g$ is added to $\mathcal{Q}$, the new set is still a restricted set.

LEMMA 6A. Suppose $t, u$ in $\mathscr{C}$ are exponentially similar and logarithmically different and $f$ in $\mathscr{C}$ satisfies $t \prec f \prec u$, then there exists $s$ in $\mathscr{C}$ such that $s, t, u$ form a restricted set and neither $f \prec s$, nor $f \succ s$ is true.

Proof. The following additional conditions need to be imposed in the inductive process used in lemma 6 which defines $\left\{\beta_{r}\right\}$. If $\beta_{1}, \beta_{2}, \cdots, \beta_{2 r-1}$ are known $\beta_{2 r}$ must satisfy (14) and (15), and in addition,

$$
\frac{\left|\log s_{2 r-1}\left(\beta_{2 r}\right)-\log t\left(\beta_{2 r}\right)\right|}{\log \log x}>r \text {. }
$$

Similarly, if $\beta_{1}, \cdots, \beta_{2 r}$ are known $\beta_{2 r+1}$ must satisfy (17), and in addition,

$$
\frac{\left|\log s_{2 r}\left(\beta_{2 r+1}\right)-\log u\left(\beta_{2 r+1}\right)\right|}{\log \log x}>r .
$$

The conditions (27), (28) will be sufficient to ensure that $s(x)$ is logarithmically different from both $t$ and $u$.

THEOREM 2A. If $\mathscr{T}$ is a countable restricted set of functions of $\mathscr{C}$, and $f$ in $\mathscr{C}$ is comparable with each element of $\mathscr{T}$, then there exists a function $g$ in $\mathscr{C}$ which is exponentially similar and logarithmically different to each element of $\mathscr{T}$, but which satisfies neither $g<f$ nor $g \succ f$.

Proof. If $\mathscr{Q}, \mathscr{R}$ are the subsets of $\mathscr{T}$ such that $t \prec f, t\rangle f$ respectively for $t$ in $\mathscr{Q}, \mathscr{R}$; the case where neither $\mathscr{Q}$ nor $\mathscr{R}$ is empty follows immediately as in the proof of theorem 2 . The case where one of the sets $\mathscr{Q}, \mathscr{R}$, say $\mathscr{R}$, is empty requires a small change in the argument.

Apply lemma $5 \mathrm{~A}$ to find an element $t_{0}$ such that $f>t_{0} \geq t$ for all $t \epsilon \mathscr{T}$ and $t_{0}$ together with $\mathscr{T}$ form a restricted set. Let

$$
h(x)=t_{0}(x) \sup _{0 \leqq \xi \leqq x} \frac{f(\xi)}{t_{0}(\xi)}, \text { for all } x>0 .
$$

Then $h(x) \in \mathscr{C}$ and $h(x) \geqq f(x)$ for all $x$. Further there will be an infinite strictly increasing divergent sequence $\xi_{1}, \xi_{2}, \cdots$ such that

$$
h\left(\xi_{r}\right)=f\left(\xi_{r}\right)_{\wedge} \quad r=1,2, \cdots .
$$

It is clear that $h$ is monotonically comparable with $t_{0}$ and so with each function of $\mathscr{T}$. We now define a function $g$, which oscillates between $t_{0}$ and $l=i^{2} h$, as follows: 
(i) let $\alpha_{1}=0, g_{1}(x)=l(x)$ for $x>0$;

(ii) if $\alpha_{1}, \alpha_{2}, \cdots, \alpha_{2 r-1} ; g_{1}, g_{2}, \cdots, g_{2 r-1}$ have been defined, we choose $\alpha_{2 r}$ to be such that

and

$$
\alpha_{2 r}>\alpha_{2 r-1}+1 \text {; }
$$

$$
g_{2 r-1}\left(\alpha_{2 r}\right)>\alpha_{2 r} f\left(\alpha_{2 r}\right)>t_{0}\left(\alpha_{2 r}\right)
$$

and define a function $g_{2 r}(x)$ by

$$
\begin{aligned}
& g_{2 r}(x)=g_{2 r-1}(x), \quad \text { if } 0 \leqq x \leqq \alpha_{2 r} \\
& g_{2 r}(x)=g_{2 r-1}\left(\alpha_{2 r}\right) \frac{t_{0}(x)}{t_{0}\left(\alpha_{2 r}\right)}, \quad \text { if } \alpha_{2 r} \leqq x
\end{aligned}
$$

(iii) if $\alpha_{1}, \alpha_{2}, \cdots, \alpha_{2 r} ; g_{1}, g_{2}, \cdots, g_{2 r}$ have been defined; we first choose $\alpha_{2 r+1}$ to be such that

and

$$
g_{2 r}\left(\alpha_{2 r+1}\right)<f\left(\alpha_{2 r+1}\right)
$$

(30) $\inf _{\alpha_{2 r} \leqq x \leqq \alpha_{2 r+1}} x^{-1}\left|\log g_{2 r}(x)-\log t_{s}(x)\right|<\frac{1}{r}$ for $s=1,2, \cdots, r$; and define a function $g_{2 r+1}(x)$ by

$$
\begin{aligned}
& g_{2 r+1}(x)=g_{2 r}(x), \quad \text { if } 0 \leqq x \leqq \alpha_{2 r+1}, \\
& g_{2 r+1}(x)=g_{2 r}\left(\alpha_{2 r+1}\right) \frac{l(x)}{l\left(\alpha_{2 r+1}\right)}, \quad \text { if } \alpha_{2 r+1} \leqq x .
\end{aligned}
$$

Here $t_{1}, t_{2}, \cdots$ is an enumeration of the elements of $\mathscr{T}$.

If we put $g(x)=\lim _{r \rightarrow \infty} g_{r}(x)$, the condition (29) ensures that $g$ is logarithmically different from $f$ and therefore from each element in $\mathscr{T}$, while the condition (30) ensures that $g$ is exponentially similar to each element of $\mathscr{T}$. The fact that $g$ is monotonically comparable with each element of $\mathscr{T}$ and that neither $g>f$ nor $g \prec f$ follows, as in the proof of lemma 6 . This completes the proof of the theorem.

Lemmas 7A, 8A may now be stated in terms of countable restricted sets $\mathscr{T}$, and the proofs are immediate. There is no lemma $4 \mathrm{~A}$, but lemma 11 together with lemma 4 will play the role of lemma $4 \mathrm{~A}$ in subsequent proofs. No change is required in lemmas 9 or 10 , so we can deduce theorems $3 \mathrm{~A}$ and $4 \mathrm{~A}$ immediately.

In the inductive definition of scales $\mathscr{S}_{\tau}$ given in section 5 , the condition (22) is now replaced by:

$$
\text { for } \mu<\tau \text {, the scale } \mathscr{S}_{\mu} \text { is a restricted set. }
$$

It will only be necessary to use theorem $4 \mathrm{~A}$ to find a function $g$, such that $g$ together with $\mathscr{T}_{\tau}$ form a restricted set and neither $g \succ f_{\tau}$ nor $g\left\langle f_{\tau}\right.$ is true, 
and put in the scale $\mathscr{S}_{T}$ all functions of the type

$$
g^{\alpha} t \text { where } \alpha \text { is real, } t \in \mathscr{T}_{\tau} \text {. }
$$

All that we need to prove is that $\mathscr{S}_{\text {T }}$ forms a restricted set. If $\alpha=\beta$, it is clear that $g^{\alpha} t_{1}, g^{\beta} t_{2}$ form a restricted set, where $t_{1}, t_{2}$ are any two elements of $\mathscr{T}_{r}$. If $\alpha \neq \beta$,

$$
\begin{gathered}
\left|\log g^{\alpha} t_{1}-\log g^{\beta} t_{2}\right|=|\alpha-\beta|\left|\log g-\log t_{3}\right|, \\
\text { for a suitable function } t_{3} \in \mathscr{T}_{\tau} .
\end{gathered}
$$

Since $g$ and $\mathscr{T}_{\tau}$ together form a restricted set, it follows that $g^{\alpha} t_{1}, g^{\beta} t_{2}$ are exponentially similar and logarithmically different and hence $\mathscr{S}_{T}$ is also a restricted set.

Thus the method used in section 5 yields

THEOREM 6. (Assuming the continuum hypothesis). There is an irreducible scale $\mathscr{S}$ which is maximal, for which any two elements are monotonically comparable, but which has neither the property $\left(\mathrm{P}_{6}\right)$ nor the property $\left(\mathrm{P}_{7}\right)$.

\section{References}

[1] Bourbaki, N., Eléments de Mathématique, Book 4, Chapter 5 (Paris, 1951), Appendix.

[2] du Bois-Reymond, P., Sur la grandeur relative des infinis des fonctions, Annali di Matematica, (2), 4 (1871), 338-353.

[3] du Bois-Reymond, P., Théorème général concernant la grandeur relative des infinis des fonctions et de leurs derivées, Jour. für Mathematik, 74 (1872), 294-304.

[4] Hadamard, J., Sur les charactères de convergence des séries à termes positifs et sur les fonctions indéfiniment croissantes, Acta Mathematica, 18 (1894), 319-336.

[5] Hardy, G. H., Pure Mathematics, 1st Edn., Cambridge (1908).

[6] Hardy, G. H., Orders of Infinity, Cambridge Tracts in Mathematics, No. 12, Cambridge $(1910)$.

[7] Rogers, C. A. and Taylor, S. J., Additive set functions in Euclidean space, Acta Mathematica, 101 (1959) 273-302.

University College, London.

The University, Birmingham, 15. 